\title{
Transformação Digital por meio de mineração e aprendizagem DevOps: Um estudo no contexto de sistemas ciber-físicos
}

\author{
Daniel D’Angelo Resende Barros ${ }^{1}$, Flávio Horita ${ }^{1}$ \\ ${ }^{1}$ Centro de Matemática, Computação e Cognição (CMCC) \\ Universidade Federal do ABC (UFABC) \\ Av. dos Estados, 5001 - Bangú 09210-580 Santo André - SP - Brasil \\ \{daniel.barros, flavio.horita\}@ufabc.edu.br
}

\begin{abstract}
Digital Transformation is a process that aims at creating more value for customers through digital tools. DevOps results in the collaboration among Development (Dev), and Operations (Ops) teams, and this alignment can make a Digital Transformation happen, encouraged by the Information Technology area. Thus, the goal of this project is creating a Digital Transformation framework through DevOps. This framework will run in cycles and, from data collected from DevOps process, will use Data Mining and Machine Learning to get relevant information, improving the Digital Transformation proposed. This framework will be validated in a cyber physical system, using continuous delivery, allowing the control of physical entities (humanoids robots).
\end{abstract}

Resumo. Transformação Digital é o processo que utiliza ferramentas digitais para gerar valor aos clientes. DevOps é o alinhamento entre os times de Desenvolvimento e Operações, e este alinhamento pode viabilizar um processo de Transformação Digital estimulado pela área de Tecnologia da Informação. Neste sentido, o objetivo desse projeto é desenvolver um framework de Transformação Digital por meio de DevOps. O framework será cíclico e, a partir de dados coletados no processo de DevOps, utilizará Mineração de Dados e Aprendizado de Máquina para obter informações relevantes e contribuir para a evolução da Transformação Digital proposta. Este framework será investigado no contexto de um sistema ciber-físico que, por meio de entregas continuas de versões, possibilitará o controle de entidades físicas (robôs humanoides).

\section{Introdução}

Com o surgimento de novos concorrentes e busca de participação no mercado, as empresas procuram constantemente serem competitivas [Berman 2012] [Van Veldhoven and Vanthienen 2019]. Clientes modernos demandam tecnologia para confiar, deslocar-se ou comprar produtos com segurança, e eles esperam as mesmas boas experiências independentemente do meio que acessam (via computador, tablet ou smartfone) [Melo et al. 2018] [Martin and Todorov 2010]. Nesse contexto, clientes exigem que seus fornecedores sejam digitais, forçando então uma busca de Transformação Digital para todas as empresas, independentemente do setor, tamanho ou tempo de mercado, liderando uma jornada de transformação de modelos tradicionais para modelos transformados digitalmente [Chanias et al. 2019]. 
Devido à familiaridade com processos e ferramentas digitais, sugere-se então que a área de Tecnologia da Informação (TI) estimule o processo de Transformação Digital (TD) nas empresas. De igual maneira, o uso de técnicas de Mineração de Dados (MD) e Aprendizado de Máquina (AM) em DevOps surge como uma importante ferramenta de apoio neste processo porque permite a entrega rápida e eficiente de versões refinadas de produtos de software. TD é um processo evolutivo que utiliza capacidade e tecnologias digitais permitindo que modelos de negócios, processos operacionais e experiências gerem valor para o cliente [Morakanyane et al. 2017]. DevOps é o alinhamento de todas as ferramentas, responsabilidades e processos na TI entre as equipes de desenvolvimento (Dev) e as equipes de operações (Ops) [Aljundi 2018]. MD é a extração de informação implícita, previamente desconhecida e potencialmente útil a partir de um conjunto de dados [Han et al. 2011]. AM pode ser definida, simplificadamente nesse contexto, como a detecção automatizada de padrões em dados [Shalev-Shwartz and Ben-David 2014].

Neste cenário, o objetivo desta pesquisa é mostrar que TD pode ser facilitada utilizando DevOps como ferramenta de apoio. Aplicando-se DevOps como processo colaborativo cíclico entre as áreas Dev e Ops, dados importantes nesse processo são coletados e classificados como experiências anteriores no processo de DevOps. Com isso, o trabalho utilizará essas experiências anteriores no processo de DevOps aplicando técnicas de MD e AM para apoiar na TD em um estudo de caso de sistemas ciber-físicos.

\section{Apresentação do problema}

Compreendido a importância da TD, surge a questão: Como realizar uma TD de modo estruturado, progressivo e eficiente? TI como uma das áreas mais inovadoras pode incentivar e, efetivamente, fazer parte da jornada de TD. Isso porque TI possui o conhecimento técnico de processos e ferramentas digitais, facilitando o início e acompanhamento da jornada de TD. Além do trabalho técnico, TI deve ainda criar um ambiente estimulante para inovação, onde novas ideias possam nascer adequadamente, casos bem sucedidos sejam aprendidos, novos processos e dados apareçam de experiências anteriores, e processos consolidados sejam rápidos e coesos. Considerando DevOps como principal ferramenta de TI, e que TI seja de fato uma das principais áreas motivadoras da TD, a meta é comprovar o vínculo estabelecido entre a TD por meio de DevOps utilizando como apoio MD e AM, a fim de compreender suas sinergias, benefícios, demandas e necessidades, tanto de pesquisa, quanto de desenvolvimento tecnológico.

A relevância dessa pesquisa encontra-se no fato de comprovar a relação de como DevOps pode ser efetivamente uma das fontes de estratégia de TD. É possível encontrar trabalhos relacionados onde o conceito de DevOps é adequadamente descrito e explicado. Outros, além da definição clara de DevOps, incluem como ser eficiente em processos relacionados [Aljundi 2018]. Adicionalmente, alguns trabalhos relacionados à TD definem o conceito com uma assertividade relativa [Morakanyane et al. 2017], e outros mostram alternativas para oferecer novas propostas de valor ao cliente, remodelando suas operações comerciais, por exemplo [Berman 2012]. Como fonte de inspiração da pesquisa, alguns trabalhos já relacionam a tecnologia como um agente de mudança, onde os gestores avaliam o papel de seus departamentos de TI para alcançar a TD [Hess et al. 2016], sugerindo um vínculo potencial entre TD e DevOps. 


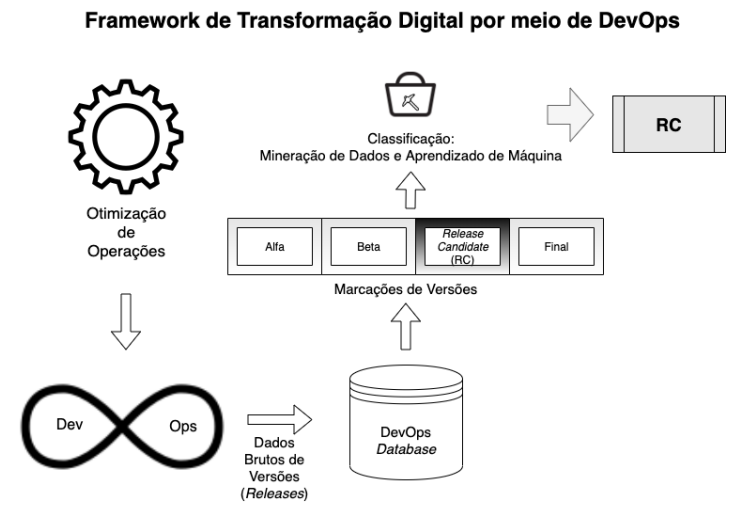

Figura 1. Transformação Digital por meio de DevOps - Abordagem RCs

\section{Proposta de solução}

Este projeto propõe como solução o desenvolvimento de um framework de TD por meio de DevOps, utilizando como fator de viabilização a entrega contínua de versões (Releases), indicados pela marcação prévia de Release Candidate (RC). Esse framework será utilizado no contexto de análise de um sistema ciber-físico que, por meio de entregas continuas de versões, possibilitará o controle de entidades físicas (robôs humanoides).

O framework, indicado na Figura 1, contextualizará a TD na principal tarefa "Otimização de Operações". Considerando um processo de DevOps de apoio, obtém no passo seguinte dados brutos de versões (número de erros, testes envolvidos, tempo de instalação, entre outros), ou seja dados não processados, dos Releases provenientes do processo de DevOps. Os dados coletados são armazenados em um banco de dados, identificado como "DevOps Database", onde será aplicada técnicas supervisionadas de MD e AM para classificação dos Releases. O framework propõe obter dados não processados relacionados aos Releases (Dados Brutos de Versões), e tem como objetivo final a informação se o Release é candidato a ir para Produção (RC) ou não.

A marcação RC indica a maturidade de uma versão para ir para Produção, levando em conta requisitos de usuários e negócios. RC indica também uma versão em Produção em breve, possibilitando calcular o tempo de uso de servidores, alocação de equipe de apoio, notificar usuários e pessoas envolvidas com o sistema e muito mais. RC, portanto, pode contribuir bastante para uma TD, levando em conta informações valiosas que essa indicação entrega. Considerando a obtenção da informação de RC em um processo cíclico de DevOps, é possível relacionar então TD com DevOps e utilizar o framework proposto para obter informações estratégicas e evoluir progressivamente a TD.

Estabelecendo esse direcionamento de obter estratégias a partir do banco de dados de DevOps, mensura-se os resultados, pois todos os ciclos de DevOps possuem início, meio e fim agendados previamente. Com isso, conclui-se que o trabalho de pesquisa pode ser baseado nas informações geradas pelos ciclos de DevOps visando o constante aprimoramento da TD.

\section{Projeto de avaliação da solução}

Para avaliação da solução, se o RC foi de fato atualizado para o controle dos robôs humanoides e qual foi o resultado esperado, serão coletados dados qualitativos e quanti- 
tativos de levantamentos e estudos de caso de interações humano-robô. Os dados serão obtidos por meio de questionários, medições e entrevistas, onde será possível observar o retorno de como foi a experiência do humano com o robô. Serão coletados também dados do banco de dados disponibilizado pelo sistema ciber-físico, onde teremos o retorno da interação na perspectiva do robô. Os dados então serão processados, padronizados em formato conhecido e armazenados em um banco de dados histórico. A partir desse banco de dados, serão aplicadas técnicas para analisar os dados e obter informações, avaliando se de fato a solução atingiu os objetivos esperados.

\section{Atividades já realizadas}

Como atividades já realizadas, um estudo prévio e validação sobre a aplicabilidade da linha de pesquisa em um contexto de sistemas ciber-físicos na empresa que o aluno trabalha em um projeto PIPE financiado pela FAPESP. Uma revisão sistemática em execução no tema de pesquisa. Um artigo em fase de aceitação e publicação, onde observa-se a classificação com assertividade acima de $80 \%$ predições de RC por meio de dados originados de DevOps, comprovando que de fato TD pode utilizar DevOps como ferramenta estratégica de evolução.

\section{Conclusão}

O objetivo da pesquisa é mostrar que DevOps, por meio de MD e AM, pode estimular uma TD eficiente. Na proposta de solução foi apresentado um framework cíclico que, a partir de dados coletados no processo de DevOps, utilizará MD e AM para classificar quando teremos um RC e contribuir para a evolução da TD proposta.

A partir de boas práticas de DevOps, espera-se termos estratégias para facilitar e alavancar a TD. Com o sucesso desse projeto, TI poderá estimular em grande parte o processo de TD e propor agendas de curto/médio/longo prazos, abrindo espaço para que a TD possa responder importantes questões sobre como a mudança pode trazer diferenciais competitivos às empresas, e como esses diferenciais podem na prática melhorar o desempenho e torna-las referência no mercado. Espera-se que o papel de DevOps na TD proporcione estratégias mensuráveis para que a etapa ou objetivo da transformação seja atendido. Por exemplo, podemos analisar o potencial retorno de informações importantes de TD por meio de uma análise mais detalhada de um banco de dados de DevOps, onde dados seriam convertidos em informações para motivar e melhorar a TD.

As futuras linhas de trabalho devem concentrar-se em encontrar novas tecnologias digitais. Estratégias de transformação usando o potencial de um banco de dados da ferramenta DevOps. Essas novas estratégias podem responder questões importantes que permitem vantagens às empresas, como quais serviços precisamos atender às necessidades dos clientes, um novo produto do portfólio disponível e muito mais. Uma vez que os dados estão disponíveis e podem ser analisados facilmente, técnicas de MD e AM podem ser usadas para responder problemas conhecidos ou novos.

\section{Agradecimentos}

Os autores agradecem ao apoio financeiro do Conselho Nacional de Desenvolvimento Científico e Tecnológico (CNPq), Processo Nro 437937/2018-6. 


\section{Referências}

Aljundi, M. (2018). Tools and practices to enhance devops core values. Master Thesis For LUT university.

Berman, S. J. (2012). Digital transformation: opportunities to create new business models. Strategy \& Leadership, 40(2):16-24.

Chanias, S., Myers, M. D., and Hess, T. (2019). Digital transformation strategy making in pre-digital organizations: The case of a financial services provider. The Journal of Strategic Information Systems, 28(1):17-33.

Han, J., Kamber, M., and Pei, J. (2011). Data mining concepts and techniques third edition.

Hess, T., Matt, C., Benlian, A., and Wiesböck, F. (2016). Options for formulating a digital transformation strategy. MIS Quarterly Executive, 15(2):123-139.

Martin, K. and Todorov, I. (2010). How will digital platforms be harnessed in 2010, and how will they change the way people interact with brands? Journal of Interactive Advertising, 10(2):61-66.

Melo, C. d. O., Figueiredo, R. M. d. C., and Venson, E. (2018). Descentralizando ti com a adoção de end-user development: dois estudos de caso. iSys-Revista Brasileira de Sistemas de Informação, 11(1):5-29.

Morakanyane, R., Grace, A. A., and O'Reilly, P. (2017). Conceptualizing digital transformation in business organizations: A systematic review of literature. In Proceedings of the 30th Bled eConference, pages 1-21.

Shalev-Shwartz, S. and Ben-David, S. (2014). Understanding machine learning: From theory to algorithms. Cambridge University Press.

Van Veldhoven, Z. and Vanthienen, J. (2019). Designing a comprehensive understanding of digital transformation and its impact. In Proceedings of the 32nd Bled eConference, pages $745-763$. 